\title{
La querella por la memoria en Édouard Glisant y Derek Walcott
}

\author{
The complaint for memory in Édouard Glisant y Derek Walcott
}

\author{
Claudia Caisso
}

Recibido: 7 de Marzo 2012. Aprobado: 23 de Abril de 2012

\begin{abstract}
Resumen
El trabajo describe el anudamiento que en el Caribe francófono y anglófono asume la querella por la memoria en las últimas décadas del Siglo XX. A partir de la lectura de varios fragmentos de $E l$ discurso antillano (2005) de Glissant en los que se argumenta a propósito del valor de la poética del acriollamiento como estrategia para transformar imaginarios impuestos por la colonialidad del poder (Mignolo, 2003) se analizan los motivos de defensa de las lenguas vernáculas con que se articula una perspectiva en que la literatura despliega su operación crítica de integración cultural. Se relaciona la descripción de los principales factores expuestos en la afirmación del Caribe como frontera y diálogo intercultural en "La querella con la Historia" (2005) de Glissant con la operación de defensa de la memoria por la literatura desplegada por Derek Walcott en "La musa de la Historia" (2000). Puesto que más allá de las diferencias que existen entre ambos escritores, comparten valiosos vasos comunicantes a propósito del destino que le asignaron a la articulación de la imaginación y el deseo de "anamnesis" en el cuestionamiento del discurso historiográfico europeo, reconocible en la confrontación de los mencionados ensayos.
\end{abstract}

Palabras clave: Interculturalidad; Caribe; Poéticas del "acriollamiento"; Memorias.

\begin{abstract}
The paper describes the knotting in the Francophone and Anglophone Caribbean takes memory complaint in the last decades of the twentieth century. From reading various fragments of the Antillean discourse (2005) in which Glissant argues with regard to the value of the poetics of creolization as a strategy to transform imaginary imposed by the coloniality of power (Mignolo, 2003) analyzed the grounds of defense of the vernacular with a perspective that literature review unfolds its operation articulates cultural integration. The description of the main factors identified in the statement as Caribbean border and intercultural dialogue in "The complaint with History" (2005) with the operation of Glissant defense memory for literature displayed by Derek Walcott relates in "The muse of History" (2000). Because beyond the differences between the two writers share valuable purpose of communicating vessels to destination assigned to the articulation of the imagination and the desire to "anamnesis" in questioning the European historiographical discourse, recognizable in confronting the above tests.
\end{abstract}

Keywords: Interculturalism; Caribbean; Poetics of "creolization"; Reports. 


\section{Desbloqueo de la memoria y "opacidad" en Glissant}

En 1976, en el Segundo Festival Carifesta organizado en Kingston, Jamaica, Edward Kamau Brathwaite leyó una presentación que habría de transformarse más tarde en el ensayo "Historia de la voz" (117), mientras que el martiniqueño Édouard Glissant llevó una ponencia titulada "La querella con la historia", después incluida en el capítulo "Historia, Historias" del célebre libro El discurso antillano (171).

Con un gesto de cortesía hacia el Caribe anglófono que todavía invita a estudiar los vasos comunicantes que las "póeticas del acriollamiento" (Phaf, 311) le imprimen al "archipiélago de fronteras externas" (Pizarro, 15), Glissant reconocía el valor de una tensión densa y fecunda. Puesto que hacía referencia allí, al profundo valor seminal que en función de las culturas del área abría la polémica sostenida entre la Historia y la literatura por una serie de creadores anglófonos -tales como el crítico Edward Baugh, el historiador Kamau Brahtwaite, antes mencionado, el narrador triniteño Vidia De Naipaul, el novelista barbadense George Lamming, y el novelista guyanés Wilson Harris-. Así como también destacaba el hecho de que el "encuentro de literaturas" antillanas -anglófona, francófona, hispanohablante, créole (171)-, lejos de obedecer a las estrategias de sus creadores respondía a los "efectos solapados" de un movimiento histórico común. Las figuras del historiador como poeta -a propósito de Brathwaite- del novelista como historiador -a propósito de Vidia Naipaul- , la historia como proyecto -en la voz de Lamming- leídas en The West Indian Writer and his Quarrel with History de E, Baugh, volvían a bordar los dilemas de la construcción de una poética de acriollamiento en vías de realización, en contrapunto conflictivo con los intereses que habían articulado la construcción de la subjetividad moderna o histórica.

En el caso puntual del Caribe, tal moral de escritura aparecía interpelada contra el telón de fondo de una constelación de producciones simbólicas que en torno de rasgos diferenciables entre el cuento antillano, la escritura de ficción y la historia, llamaban a poner en relación a las culturas letradas con las orales a los efectos de hacer tomar partido por las últimas hasta incluir sus rastros en la transformación de la ficción escrita. Así como también invitaban a reconsiderar la situación de inexistencia a la que estaba condenada la

Poligramas 37, primer semestre 2013, ISSN 0120-4130. 
memoria colectiva en consonancia con las huellas irreductibles de la "opacidad" antillana. Opacidad de la lengua creóle y de los fenómenos culturales que según Glissant remiten a una vasta operación de reivindicación de las estrategias de resistencia de poblaciones marcadas por la diáspora, destinadas a encontrar la alienación en la asimilación a la cultura del Otro hegemónico, al tiempo que la inquietante experiencia de resistencia. Opacidad, entonces, cuyo estatuto ambiguo irrumpe como una barrera frente a la transparencia de la razón universal y admite ser leída como un rasgo de desposesión del hombre común que no puede ejercer la conciencia acerca de su identidad, pero que simultáneamente significa un valioso enigma. Puesto que suscita la necesidad de observar la potencialidad que concede la posibilidad de "vivir" en un espacio en el que la ausencia de raíces prodiga el "devenir" antillano a condición de interrogar los atributos con los que el hombre europeo lo ha “encubierto". Movimiento abierto por Glissant por el que desplazando y transformando el concepto poético del "retour" trazado por Aimé Césaire en el poema fundante de la negritud "Cuaderno de un retorno al país natal" (1939) (Figueiredo, 40), se describen las prácticas de rodeo en la vida cotidiana y al rodeo mismo, como la única vía plural de abordaje de las culturas del Caribe. Rodeo que es posible reconocer en el créole como operación de desestabilización y al mismo tiempo de fisura de la "pulsión mimética" que, desde Frantz Fanon a Derek Walcott, aparece como matriz de singularización de la compleja pluralidad del área.

El rodeo oficia en Glissant de enlace entre la búsqueda de "huella primordial” y la relación: apertura a lo abierto del mundo desde el reverso de una totalidad hegemónica, agresiva y eurocentrada. Que como impulso implica tanto una réplica al movimiento de uniformidad que instaura la globalidad moderna como un desplazamiento del movimiento del retorno al África propuesto por la negritud en su profundo enfrentamiento del hombre occidental por las atrocidades perpetradas por la "trata".

Materialización de una suma o acumulación de fragmentos y parlamentos para nombrar al sesgo "lo real antillano", cuya unidad es menos el sueño de una unidad política en el sentido cabal del término, que la utopía de la comunidad cultural sostenida sobre el asiento del habla y el amalgamiento de las lenguas (Figueiredo, 39). 
Glissant describía momentos de las producciones culturales caribeñas en las que el lugar de enunciación no sólo produce imaginarios relevantes, porque atañen a la vida de todo el planeta sino que exponen vivencias de una geografía fronteriza que en sus singulares derivas ponen en cuestión el relato de la transparencia universal. Práctica de las culturas caribeñas que en su dimensión periférica hacen señas sobre circunstancias de un presente que no admite la objetivación, como tampoco admite la reflexión sobre el "ser" antillano en función de la experiencia de la cosificación.

En la producción de grietas (43-56) y distancias más o menos amables respecto de la negritud, exponía al Caribe como un genuino laberinto (Grüner, 469) sin renunciar al riesgo de desplegar un discurso signado por la oscuridad. Para que la opacidad de los antillanos fuera proclamada como un derecho conquistado a fuerza de vivencia ante la imposibilidad de definir "lo" antillano" abriendo cauces de trabajo en el deseo de metáfora y la "intención poética" como sustitutos constantes de la intencionalidad histórica. Una "poética de la relación" que implica celebrar el acontecimiento de las lenguas consideradas menores para tender puentes entre lo local no genocida y lo global en un gesto en el que indefectiblemente se precipitan la gesta heroica, la jerarquización de los idiomas para pensar, y la exaltación del ego. Desde el ego del conquistador hasta el ego poético que irrumpe en las llamadas "poéticas del uno" (283), poéticas exaltadoras de la singularidad subjetiva que a veces conducen según Glissant a "lo indecible poético" (277) y/o a la fijeza del arquetipo. Ya que en varios momentos de El discurso antillano es posible reconocer un movimiento de "irrisión" (158) de la subjetividad creadora de la literatura europea canónica hasta que el Caribe es concebido como un "entre lugar" (Pizarro 2002, 28): la América Otra o el estuario de las Américas. Un sitio de desposesión de la historia en el cual la consideración de los fenómenos de “diversalidad” o de descastamiento del lugar del Amo, en particular a propósito de la relectura de un "texto pórtico" (Jáuregui, 461) para el Caribe como es La tempestad de Shakespeare, se transforma en decisivo.

¿Qué es esa legitimidad en Shakespeare -anota Glissant-, sino la sanción del equilibrio naturaleza-cultura, con la cual el hombre abandonaría las antiguas alquimias unificadoras de la Edad Media y entraría en la energía diversificada de lo que se llamará tiempos 
modernos? (Grandiosa perspectiva, que nos da la medida de la obra shakesperiana, con (desafortunadamente) un elemento desapercibido: en el equilibrio-totalidad se ha instalado una jerarquía, de Calibán a Próspero; y no resulta difícil ver que Calibán-naturaleza se opone desde abajo a Próspero-cultura.Así, en La tempestad, la legitimidad de Próspero está vinculada a su superioridad y se convierte en legitimidad de Occidente. La ambigüedad es entonces que en Occidente la literatura y la historia se hayan propuesto actuar al mismo tiempo en la Totalidad (voltear la linealidad primitiva en globalidad), pero que en esta totalidad postulada se haya insertado la inaudita ambición de animar al hombre a imagen y semejanza del occidental, con diversos grados de elevación, de Calibán a Próspero” (188189)

No por casualidad como señala Eurídice Figueiredo (46) muchos escritores caribeños reescribieron La tempestad para invertir el anatema. Al hacer coexistir la Historia con las historias Glissant despliega la transversalizacíon, como forma genuina de interacción discursiva. Transgrede la construcción jerárquica de la totalidad eurocentrada a la que el discurso historiográfico ha contribuido de manera ejemplar. Orienta la reivindicación del cúmulo de prácticas que desde los relatos sostenidos por el contador de historias oral hasta las operaciones de di-versión de los usos del créole hacen señas sobre la construcción de una poética para "lo real antillano". En la que ese real requiere de la comparación y diferenciación de las culturas letradas y las culturas orales. Ya que es precisamente la marca del "deseo histórico" que está en la novela y no en la historiografía, la que según Glissant permite especificar las formas de supervivencia de las culturas populares antillanas cuando se destacan los logros y los fracasos a propósito de cada una de esas intervenciones. Puesto que la revisión de ciertos horizontes de escritura reconocibles en Absalóm, Absalóm de William Faulkner, Cien años de soledad de Gabriel García Márquez y Los pasos perdidos de Alejo Carpentier (198-207) le permiten argumentar sobre el valor de la novela contemporánea con la aporía de desmesura que aquélla juega en su intento por relevar al mito. Mientras que el relato oral en créole carece de aquel sentido de lo sagrado, como carece de datación y de búsqueda de trascendencia. A expensas de la descripción de aquella escena irrumpe la representación del trazo primordial que moviliza el deseo histórico como

Poligramas 37, primer semestre 2013, ISSN 0120-4130. 
vector irreductible tanto en la novela, la memoria colectiva como en el diapasón del mito a propósito de la "visión profética del pasado" (175). Lo que está ligado visionariamente a medias con lo que sucedió y lo que no sucedió y sin embargo implica un cosmos que no requiere de ninguna prueba. El territorio de lo irrepresentable cuya particularidad "es obscurecer al revelar" (Glissant, 198), en el que las marcas elusivas y fragmentarias de la narración contemporánea abren su diferenciación y similitud en contrapunto con las formas de simbolizar de las culturas orales, en particular las africanas. Puesto que la imagen de la "visión profética del pasado" que Glissant usa a veces para potencializar el lugar de la ficción narrativa y teatral frente al discurso historiográfico, admite ser traducida como el margen de una exploración que es obsesión de escritura, y dramatización a la hora de desacralizar los "efectos" de marcha de la causalidad histórica. Entre la experiencia de la totalidad narrable de la epopeya contada por los griots africanos -“en la poética del texto oral africano, todo es decible" escribe (277)-, la naturaleza parcelaria del cuento antillano en el que no es posible concebir al tiempo como dimensión fundante del hombre (204) y la pérdida de objetividad y de realismo canónicos de la novela contemporánea, Glissant dibuja los rodeos de una economía diferente para la palabra literaria con que erosionar la trama y la trampa de la Historia. Apuesta a la diferenciación de prácticas simbólicas que en el espacio de la comparación arrojan caracterizaciones de índole antropológico. Si la novela fracasa en su intento de relevar el lugar del mito, el cuento popular créole fracasa en la posibilidad de transformar su dimensión de "poética forzada" en poética creativa o "natural" -signada como está por la colonialidad impuesta en la economía de plantación(260). No obstante en su fracaso ofrece el despliegue de "arranques sacrílegos", que en la mirada de Glissant, contrarrestan la sacralidad de lo escrito en Occidente (204). Puesto que la objetividad del narrador de la Historia y la voluntad de realismo decimonónico o "clásico" son desidealizados hasta que se los declara vanos de toda vanidad en su pretención de describir lo real(187), en particular: lo real antillano. Filo que insistentemente modula reflexiones contrastadas entre la orilla del Caribe y del África, o de las Américas, África y Europa y que es presentado en términos de un derrotero por lo "conocidodesconocido" (184), o como “perversión del lugar de la filiación y del origen” (200), 
trabajado a propósito de juegos antitéticos, comparaciones y diferenciaciones que permiten contradecir la "doble aspiración de una Historia con $\mathrm{H}$ mayúscula y de una literatura sacralizada en el absoluto del signo escrito" (190).

Tal construcción de "querella con la Historia" irrumpe en las reflexiones de Glissant según ya se ha señalado- a la luz de una "deuda" simbólica explícita con el Caribe anglófono que permite recorrer la imaginación materialista de un "renacimiento" etnorracial. Sin embargo aquel reconocimiento de la fuerza de religación que han propiciado las "culturas literarias" a contrapelo de las lógicas coloniales - como intervención reactiva ante los relatos metropolitanos y los de los antillanos fascinados por aquéllos- nunca se consuma como gesto fundador. Así en "La querella..." Glissant escribe: Las Antillas son el lugar de una historia hecha de rupturas y cuyo inicio es un arrancamiento brutal, la trata de negros. Nuestra conciencia histórica no podía "sedimentarse" -por así decirlo- de forma progresiva y continua como en los pueblos que engendraron una filosofía de la historia a menudo totalitaria -los pueblos europeos- sino agregarse, por efectos del impacto, de la contracción, de la negación dolorosa y la explosión. Esta discontinuidad de lo continuo, y la imposibilidad para la conciencia colectiva de circunscribirlo, caracterizan lo que yo llamo una no-historia (172).

De esa herida habla la falta de recuerdo que según el autor existe en la región a propósito del arco "Matouba-Moncada" (174) abierto entre el episodio de resistencia heroica sostenida por el general Delgrés - de origen martiniqueño- y el cubano Fidel Castro. Pero de esa herida también hablan la búsqueda de una economía otra para las palabras, donde fuera posible oír y hacer escuchar los conjuntos de las historias di-fractadas. Desde la opacidad que en situaciones de diglosia el créole sostiene en relación con la lengua francesa, cuando una lengua permanece amenazada por la otra, y sin embargo sugiere la posibilidad de crear utopías de coexistencia, se abre el campo de las reminiscencias que afirma el juego por el que el deseo de anamnesis sustituye la tentación unívoca de hegemonía política. La querella de Glissant enfatiza así el rostro neurótico de la búsqueda de explicación histórica mientras cifra la transversalidad anehlada de saberes en la metáfora de la "unidad submarina" de Brathwaite. La huella productiva de lo imposible, como ha sido productivo lo posible del 
relato trabajado en los límites de la novela escrita en su cotejo con el relato épico africano, o el cuento oral antillano. En términos de "raíces submarinas: es decir derivadas, no implantadas con un solo mástil en un solo limo, prolongadas en todas las direcciones de nuestro universo por su red de ramas" (178). Habiéndose autorizado en el pensamiento analítico de Derek Walcott cuando señala que la historia como conciencia de la acción y como vivencia (176) no es sólo asunto de los historiadores.

\section{Derek Walcott a contrapelo del 'cinismo metropolitano'}

¿Era posible crear algo genuino, se preguntaba Walcott en el incisivo artículo “Cultura o imitación?" de 1974 para responder al desencanto expuesto por Naipaul en The Middle Passage..." (1962) respecto de que nada podía crearse en el Caribe?. ¿Qué era necesario decir para hablar de las tensiones y los deseos en un espacio en el que la imitación estaba tan íntimamente ligada a la supervivencia?. ¿Hasta dónde podía argumentarse sobre el valor irreductible de las culturas caribeñas lejos del camouflage y la propalación de unos espejos que no cesaban de mostrar la destrucción trágica del original?.

En el Caribe -escribía Walcott- la historia es irrelevante, no tanto porque no se esté haciendo, o porque haya sido sórdida; sino más bien porque jamás ha importado. Lo que ha importado es [...]la amnesia de las razas [...]El epitafio del Sr Naipaul sobre el inmenso esfuerzo inútil de las Indias Occidentales no ha abortado, sin embargo, la pasión con que la cultura de las Indias Occidentales continúa procreando el mimetismo, porque la vida, si podemos hablar de eso en el archipiélago, despiadadamente lo continúa (Hamner, 53) ${ }^{31}$.

En el ardid endémico del señuelo al que apelan como herramienta de defensa los lagartos y los camaleones, en los procedimientos de imitación sostenidos por los monos y los loros, Walcott dejaba traslucir el dilema por medio del cual era tan inútil pensar en una literatura del Caribe autónoma del Viejo Mundo, como encomiable esperar que se alejara de la discriminación racial, la desesperación y la venganza. El gesto, destinado a mostrar las estrategias de supervivencia del Caribe afirmaba la apuesta por la ficción que desde la colonialidad anhelaba cuestionar el "cinismo metropolitano" (79). Más allá de la afición

\footnotetext{
${ }^{31}$ La traducción y el subrayado son nuestros.
} 
por el exotismo que era fácil advertir en los relatos de los viajeros del siglo diecinueve, del racismo del historiador Froude -a quien Naipaul seguía- y de la falta de protagonismo de las poblaciones del Caribe en la construcción de su destino, Walcott insistía en el hecho de que efectivamente nada ni nadie podía ser creado, por el grado de extraordinaria excepcionalidad de lo que allí irrumpía. Desde el carnaval antillano hasta el paisaje con su contundente presencia invitaban a interrogar "el ejercicio de la memoria racial que es la tradición" (Hamner, 53) ${ }^{32}$, y a señalar que el Caribe era el otro lado del espejo de la civilización europea al que ya no se podía regresar. Ya que si se podía regresar a algún lugar, era adonde sólo hubiera "simulaciones de auto-descubrimiento" (Hamner, 53) ${ }^{33}$.

En el ensayo también decisivo que lleva por título "La musa de la Historia" (1974) Walcott insistía en destacar que en los grandes poetas adánicos de la América continental, desde Whitman a Neruda, era posible reconocer el hallazgo de los trazos del hombre del Nuevo Mundo por el rechazo que esos creadores hacen de la visión de la historia como avidez por la identidad cuando la lengua es la mejor oportunidad para esclavizarse. Walcott buscaba una suerte de empatía con escritores continentales que le permitiera desviar la mirada sobre los modelos europeos con que construir diferencias en el Caribe, así como también quebrar la idea de un pasado estático. Pero en el contrapunto se encontraba irremediablemente con las marcas locales de la amnesia y con la imposibilidad de concebir a las Antillas como un Edén. Si en Whitman y Neruda podía reconocer la construcción valiosa de un hombre nuevo a propósito de que sus voces no se ponían al servicio del discurso histórico, no le resultaba sin embargo fácil asimilar sus posiciones estéticas con lo que intuía debía articular una suerte de "Paraíso sin historia", cuya ética adánica fuera a contracorriente de lo más evidente para celebrar lo caribeño raigal, vital y local, todavía más allá del registro legendario que celebraba en los Elogios de Saint John Perse y en el Cuaderno de un retorno al país natal de Aimé Césaire.

El Caribe como frontera fracturada del Imperio insistía en recordarle que no era allí donde el Imperio terminaba, sino más bien donde habría de revelarse, y que para "ser portadores de un mensaje" (47), para evitar "afirmaciones de identidad forzada" (42) se trataba de

\footnotetext{
32 íd. anterior.
}

33 íd. anterior.

Poligramas 37, primer semestre 2013, ISSN 0120-4130. 
reconocer que lo que se necesitaba, no eran "nombres nuevos para cosas antiguas, o nombres antiguos para cosas antiguas -según ya había dicho en "La voz del crepúsculo" sino la fe necesaria para usar de nuevo los nombres antiguos" (21).

El giro implicaba construir el dispositivo de una compleja co-existencia entre el descubrimiento de las ruinas del presente y una vuelta al pasado alentada por la valoración de las tradiciones europeas junto a la representación de la vida extrema puesta en escena por la danza y el ritual.

Walcott reproduciría y variaría una y otra vez las lecciones del imaginismo americano a través de las lecturas de Pound y de Eliot con el recurso de volver a Dante a la manera de un genuino maestro generado en la advertencia del fracaso de la civilización occidental, cuyas teorías sobre el italiano, en términos de dialecto nuevo y más potente en relación con el latín imperial de la antigua Roma, le brindarían valiosas iluminaciones y procedimientos para eludir problemas de representación relativos al experimentalismo y la abstracción inherentes al arte contemporáneo. En un paso que perspectivizada la empatía entre el Caribe y el Egeo considerando al primero como una suerte de "Nuevo Egeo". Así como formulaba el "empate" entre el creole antillano y los dialectos que precedían la afirmación de los idiomas nacionales en la alta Edad Media europea. Tránsito hacia el cual Walcott exponía desde sus tempranos trabajos el mapa del Nuevo Mundo en analogía abierta entre los héroes homéricos, marinos y pescadores como es posible reconocer en el tratamiento que el tema del "viajero afortunado" conquista en "La goleta el vuelo", en El reino del caimito .

Junto a ese complejo proceso registrado en sus reflexiones, entrevistas y textos literarios, resguardaba férreamente la lección que le ofrecían las culturas populares antillanas a través del vasto ritual de sus performances con la capacidad de conservación y transmisión de la memoria que la intervención del contador de historias orales manifestaba (35). En el amalgamiento de genealogías culturales letradas y populares, Walcott dejaría ver a lo largo de más de cuarenta años, una ardua labor para reconciliar las herencias riquísimas que habían sido enfrentadas por la colonialidad del poder. Una búsqueda de resonancia de un arte cuyos alcances fuera capaz de concitar la intuición y el asombro ante la experiencia

Poligramas 37, primer semestre 2013, ISSN 0120-4130. 
perdida, y que requería de creencia y trabajo para dislocar la disimetría existente entre el archipiélago y la metrópolis.

El auténtico cuento popular [...] había surgido -según se lee en "La voz del crepúsculo"en otro tiempo, al amor de la lumbre o en la puerta de la choza, cuando la noche, en el exterior, era una fuerza hostil, infestada de diablos, de demonios de los bosques, un espacio para que el alma emprendiese su viaje, y cualquier niño que hubiera escuchado esa simetría cantada querría reproducirla intacta cuando le llegase el momento de convertirse en su propio contador de historias. El aparente conservadurismo de la ficción antillana, tanto en la narrativa como en el teatro, no es mera imitación de antiguos moldes, sino memoria de esa forma primitiva (35) ${ }^{34}$.

Urgía que el teatro, la narrativa y la escritura poética, con capacidad para curar la alienación cultural en sociedades en las que es sumamente complejo responder a la pregunta por la identidad, estuvieran destinados a iluminar rememorando antes que a entretener. Así como urgía que esas producciones abrevaran en las fuerzas contradictorias del Caribe con que reinventar el pasado en una apuesta diferente respecto del discurso histórico, que cumplía con intereses políticos específicos, o no era capaz de captar ni de transmitir el dinamismo de ese pasado con sus caudales de horror, seducción y complejidad. Visiones estáticas, fuerza opresiva de la finalidad, claridad y causalidad desinvertían al lenguaje, según Walcott, de la alteridad que le concedían, en cambio, el poder del "eco", el "frenesí", y la pasión por nombrar como si ellos fueran por vez primera el grito de anuncio de un lenguaje en estado naciente.

Con ácida ironía eximía a la Historia de poder inspirador y a los conquistadores y descubridores de la hazaña de descubrimiento, mientras acentuaba la fuerza del trabajo por encima de la inspiración. Los grandes poetas adánicos del Nuevo Mundo, según decía, no estaban 'atormentados por la presión del pasado sino por el peso del presente' (57). Concederle fuerza de rememoración a la ficción literaria, no sólo se asentaba, entonces, en la desconfianza respecto de las técnicas y las verdades narradas por la Historia, o en los límites del teatro vanguardista europeo al que veía ritualizado por el deseo de absolución

\footnotetext{
${ }^{34}$ El subrayado es nuestro.
} 
(desde Jarry hasta Artaud) (16), sino también en la posibilidad de construir una moral creativa con la carga de responsabilidad que ella requiriera a favor del pueblo caribeño y que fuera sustitutiva de las representaciones esquemáticas

A medida que envejecemos como raza - escribe Walcott- cobramos mayor conciencia de que la historia es algo escrito, de que es una literatura desprovista de moral, de que el ego de la raza es indisoluble y de que todo depende de si escribimos esta ficción desde la perspectiva del héroe o desde la perspectiva de la víctima (54) ${ }^{35}$.

Al caracterizar al discurso histórico como una convención Walcott concluía en el hallazgo de que la labor interpretativa que se derivaba de él configuraba una lógica binaria de valores que debía ser trascendida con la defensa de una sensibilidad urdida en la poética del híbrido y la mixtura. A expensas de un dispositivo montado sobre la reivindicación de la militancia por el arte desde donde reelaborar las tensiones existentes entre diversas vertientes de la literatura antillana, entre las que visualizaba el riesgo del aislamiento y el exotismo - propiciada por exaltadores tardo-románticos de la raza negra-, la inscripción del lugar del bastardo, facilitaba la integración vitalista de diferentes legados y conquistaba rasgos positivos a expensas de los que era posible reivindicar un nuevo lugar. Sostenía la mezcla de culturas puesto que permitía transgredir los ideales de pureza de la raza blanca como las apuestas de enfrentamiento directo a las potencias coloniales impulsadas por la negritud. La bastardía irrumpía así como un factor que podía integrar lo que la Historia en el sentido de memoria colonial y de proceso de racialización había separado porque el "mulato del estilo" es quien puede traicionar el dualismo maniqueo que la lógica de la Modernidad funda a propósito de la relación dura entre civilizados y bárbaros, héroes y víctimas (20,54). En tanto el bastardo puede escapar a la lógica causalista que es finalmente la de la víctima o la del héroe, es el que puede sostener el sueño de equilibrio, y el gozo de la posibilidad en la imprevisiblidad (59). La serie del bastardo, el mulato, o el mestizo trabajada en los textos walcottianos donde se abordan cuestiones de vida y cultura caribeñas, abre la fisura por donde se fuga la supuesta pureza de origen, donde se

\footnotetext{
${ }^{35}$ Íd. nota anterior.
} 
reconstruyen el lugar del intelectual caribeño y latinoamericano, así como el redireccionamiento de la fuerza de ciertas retóricas de poesía visionaria (70) .

\section{Bibliografía}

Benavente Morales, Carolina "El original y su traducción: Edouard Glissant y Michael Dash". [on line: www.usach.cl/revistaidea]

Brathwaite, Kamau La unidad submarina. Ensayos caribeños. La Plata: katatay, 2010.

Breslin, Paul Nobody's Nation. Reading Derek Walcott. Chicago: The University of Chicago Press, 2001.

Dash, Michael "Prólogo" en El discurso antillano. La Habana: Fondo Editorial Casa, 2010.

Figueiredo, Eurídice “Construcciones identitarias. Césaire, Glissant, Chamoiseau” en Pizarro, Ana (comp.) El archipiélago de fronteras externas. Santiago de Chile: editorial Universidad de Santiago, 2002.

Glissant, Edouard El discurso antillano. Caracas: Monte Ávila, 2005.

Grüner, Eduardo La oscuridad y las luces. Capitalismo, cultura y revolución. Bs.As.: edhasa, 2010.

Hamner, Robert Critical perspectives on Derek Walcott Washington: Three Continents Press, 1993.

Jáuregui, Carlos Canibalia.Canibalismo, calibanismo, antropofagia cultural y consumo en América Latina. Madrid: Iberoamericana Vervuert, 2008.

Mignolo, Walter Historias locales/diseños globales. Colonialidad, conocimientos subalternos y pensamiento fronterizo. Madrid: ediciones akal, 2003.

Phaf-Rheinberger (ed.) "El lenguaje-nación y la poética del acriollamiento. Una conversación entre Kamau Brathwaite y Édouard Glissant”. Literatura y lingüística 19. 2008. 311-329.

Pizarro, Ana "El archipiélago de fronteras externas" en Pizarro, Ana (comp.) El archipiélago de fronteras externas. Santiago de Chile: editorial universidad de santiago, 2002.

Poligramas 37, primer semestre 2013, ISSN 0120-4130. 
Rodríguez, Jorge Emilio "Oralidad y poesía: el acriollamiento de la lengua inglesa en el Caribe" en Pizarro, Ana Palavra, literatura, cultural. Vol. 3. "Vanguarda e Modernidade".Campinas: Editora da Unicamp, 1995.

Walcott, Derek La voz del crepúsculo. Madrid: Alianza, 1998

Sancholuz, Carolina "Relecturas del Caribe: de los planteos de Édouard Glissant y Antonio Benítez Rojo al "Archipiélago de fronteras externas" de Ana Pizarro" en Sancholuz, Carolina Mapa de una pasión caribeña. Lecturas sobre Edgardo Rodríguez Juliá . Bs.As.: Editorial Dunken, 2010. 